\title{
Editorial
}

\section{Impact Welding of Materials}

\author{
Ivan Galvão ${ }^{1,2, *(\mathbb{D}}$, Altino Loureiro ${ }^{1}$ and Ricardo Mendes ${ }^{3}$ \\ 1 University of Coimbra, CEMMPRE, Department of Mechanical Engineering, Rua Luís Reis Santos, \\ 3030-788 Coimbra, Portugal; altino.loureiro@dem.uc.pt \\ 2 ISEL, Department of Mechanical Engineering, Polytechnic Institute of Lisbon, \\ Rua Conselheiro Emídio Navarro 1, 1959-007 Lisboa, Portugal \\ 3 University of Coimbra, ADAI, LEDAP, Department of Mechanical Engineering, Rua Luís Reis Santos, \\ 3030-788 Coimbra, Portugal; ricardo.mendes@dem.uc.pt \\ * Correspondence: ivan.galvao@dem.uc.pt; Tel.: +351-239-790-700
}

Received: 10 December 2020; Accepted: 12 December 2020; Published: 14 December 2020

\section{Introduction and Scope}

Recent industrial criteria, focused on obtaining increasingly efficient structures, require the production of multimaterial components. However, the manufacturing requirements of these components are not met by conventional welding techniques. Alternative solid-state technologies, such as friction or impact-based processes, must be considered. Impact welding processes have the advantage of presenting a very short cycle time, which minimises the interaction of the materials under high temperature. This fact strongly contributes to reducing the formation of brittle intermetallic compounds (IMCs), i.e., one of the main concerns of welding dissimilar materials. Moreover, as the influence of the welding process is confined to a very narrow band around the materials interface, similar and dissimilar welds with high-strength bonding and a minimal heat-affected zone can be produced.

The impact welding family encompasses different welding processes, such as explosion welding, magnetic pulse welding, vaporising foil actuator welding, and laser impact welding. Although these processes share the main operating principle, consisting of a high-velocity collision between a flyer and a target, they differ in the way the flyer is accelerated. These processes also present very different length scales, providing the impact welding family with a broad applicability range. The technical interest of impact welding is driving the ongoing development of many scientific studies, which are essential to optimise the current manufacturing processes by developing new welding strategies and solutions. The present special issue presents a sample of the cutting-edge research that is being conducted on the multidisciplinary field of impact welding.

\section{Contributions}

Eleven research papers and one review paper have been published in the present special issue. Different research approaches, i.e., experimental, numerical, and coupled experimental/numerical, are presented in the published papers. These studies, which were developed by some of the most relevant research groups working in impact welding, encompass a large spectrum of welding processes, such as explosion welding, magnetic pulse welding, vaporising foil actuator welding, and laser impact welding. Detailed analyses on the impact welding macro and microscopic phenomena, metallurgy, mechanical behaviour, numerical modelling and simulation, process developments, and industrial applications are presented in this group of works.

Six research papers on explosion welding have been published. Émurlaeva et al. [1] conducted a Smooth Particle Hydrodynamics (SPH) simulation work to further analyse the suitability of the classical approaches defining the boundaries of the weldability window. The numerical simulation results, 
which were applied to 6061-T6 aluminium alloy, were found to reproduce the basic phenomena typical of high-velocity impact welding, such as the jet and wave formation and the material deformation near the interface. The left, right, and lower boundaries of the weldability window found by numerical simulation were in good agreement with those resulting from Wittman's and Deribas's approaches. However, significant differences were found for the upper limit. In turn, a coupled experimental/numerical work focused on studying the microstructure and the mechanical properties of titanium/aluminium explosion welds before and after being submitted to heat treatment was developed by Mahmood et al. [2]. According to the authors, aluminium-titanium IMCs were formed at the weld interface, but these phases did not affect the weld strength. The authors also reported that the weld strength decreases after heat treatment due to changes in the structure of the weld interface. The SPH-based numerical simulation analysis conducted in this research was found to meet the experimental results. For example, the predicted temperatures for the weld interface were higher than the melting temperature of the alloys, which agreed well with the formation of aluminium-titanium IMCs.

Paul et al. [3] studied the microstructural and mechanical properties of tantalum/copper/stainless steel explosion-welded composites. An exhaustive experimental work, in which several characterisation techniques were used, such as scanning electron microscopy, energy dispersive spectroscopy, transmission electron microscopy, electron backscatter diffraction, microhardness testing, and bending testing, was conducted to characterise the tantalum/copper and the copper/stainless steel interfaces. The conditions experienced at the weld interface, such as severe plastic deformation, localised melting, and materials interaction, were deeply discussed based on the microstructural features and the mechanical properties of this region. An experimental work was also conducted by Carvalho et al. [4], which was focused on two different material combinations. Specifically, these authors analysed the coupled effect of two strategies for optimising the production of aluminium/carbon steel and aluminium/stainless steel explosion-welded clads, i.e., the use of a low-density interlayer and the use of a low-density and low-detonation velocity explosive mixture. The low values of collision point and impact velocities achieved for the welding tests provided the production of joints with sound microstructure and good mechanical behaviour, making the differences in weldability of both material combinations less significant. The low-detonation velocity, low-density, and ability to detonate in small thicknesses of the explosive mixture make it very suitable to be used for welding very thin flyers and dissimilar material couples that easily form IMCs.

Inao et al. [5] joined a very thin aluminium plate to two different magnesium alloys (AZ31, $\mathrm{Mg}_{96} \mathrm{Zn}_{2} \mathrm{Y}_{2}$ ) by explosion welding, using a gelatine layer as a pressure-transmitting medium. The lower energetic conditions associated with the use of a very thin flyer and the gelatine medium promoted better bonding quality than in conventional explosion welding. The welds achieved under these conditions presented a uniform, smooth surface and an interface without intermetallic-rich layers. In turn, Nishi et al. [6] conducted a work highly focused on the development of new heat-exchanger solutions. Specifically, the authors addressed the manufacturing procedure of two types of copper/stainless steel composite UniPore structures. Two specific arrangements of thin copper and stainless steel pipes were successfully compacted/welded by explosion. They were found to present excellent interfacial bonding between the component walls.

Using a different welding process from the previous works, i.e., vaporising foil actuator welding, Su et al. [7] produced aluminium/stainless steel joints with an aluminium interlayer. The authors tested different input energy values and reported that higher energy values promoted welds with higher tensile and shear strengths because of the larger weld areas obtained under these conditions. Regarding the interface morphology, it was found to be different for both interfaces. While the flyer/interlayer interface (aluminium/aluminium) was wavy, the interface between the interlayer and the baseplate (aluminium/stainless steel) was composed of continuously or intermittently distributed IMCs.

The work developed by Wang and Wang [8] was focused on characterising the flyer velocity in laser impact welding, which was measured with Photon Doppler Velocimetry (PDV) under different 
experimental conditions. The influence of the laser energy and the flyer thickness on laser energy efficiency was analysed. Other aspects, such as the standoff working window, the rebound behaviour of the flyer, and the effect of the flyer size and confinement layer on the flyer velocity, were also addressed in this research.

A study focused on magnetic pulse welding was conducted by Emadinia et al. [9]. These authors studied the influence of the surface preparation on the interface properties of aluminium/copper tubular joints. They reported that the weld quality was influenced by the surface preparation of the copper target. From the microstructural and mechanical study of welds produced with different surface preparations, it was observed that the highest weld strength values were registered for a threaded copper target, being achieved without the interfacial waviness or IMCs.

Bellmann et al. [10] studied the formation of a cloud of particles during impact welding, its characteristics, and its influence on weld formation. A detailed experimental plan was implemented by the authors, who carried out impact welding tests on different setups. These tests were monitored using a high-speed camera, accompanied by long-term exposures, recordings of the emission spectrum, and an evaluation of the interaction of the cloud of particles with witness pins made of different materials. The interfacial phenomena were discussed based on the pressure conditions, the welding parameters, the base material properties, and the surface state of the welded materials. The authors provided an estimation of the temperature reached in the joining gap. In the sequence of this work, the influence of the process parameters on the main phenomena and mechanisms of bond formation in impact welding was addressed by Niessen et al. [11]. An in-depth experimental research was presented, in which different welding setups were studied. The processes were monitored by several techniques, such as high-speed imaging, PDV, and light emission measurements. The welds were experimentally characterised by ultrasonic inspection, metallographic and microstructural analyses, and mechanical testing. This study made it possible to better understand the influence of different process parameters on the weldability window, and consequently, on the weld characteristics and properties, and to predict different bonding mechanisms.

As reported above, a review work on high-velocity impact welding was also published in the present special issue. This study, which was developed by Wang and Wang [12], presents a global overview of different impact welding processes, such as explosion welding/gas gun welding, magnetic pulse welding, laser impact welding, and vaporising foil actuator welding, and explains the formation of the jet phenomenon during these processes. The macro and micro characteristics of the bonding interface of the impact welds were also addressed at the second stage of this research. Finally, the authors studied the welding parameters, specifically, their selection and their effect on wave formation and weld mechanical properties.

\section{Conclusions and Outlook}

An encompassing view of the advances that are being made in impact welding was presented in this special issue. This was a consequence of the quality and diversity of the published papers, in which highly supported experimental, numerical simulation, and review researches on a broad spectrum of impact welding processes were presented. However, the research in impact welding is far from being saturated, and strong research is still necessary so that the industrial applicability of this family of processes can grow in the future.

As the Editors of the present special issue, we consider that it was a very successful project, which made us especially happy. However, it would be impossible for this special issue to succeed without the valuable contributions of all the authors. They were the main actors of this project and we are profoundly grateful to all of them. We also want to thank all the reviewers, whose work was crucial for ensuring the quality, scientific relevance, and actuality of the published works. Finally, we acknowledge all support provided by the Metals editorial team, especially by Kinsee Guo, during the development of this special issue. 
Conflicts of Interest: The authors declare no conflict of interest.

\section{References}

1. Émurlaeva, Y.Y.; Bataev, I.A.; Zhou, Q.; Lazurenko, D.V.; Ivanov, I.V.; Riabinkina, P.A.; Tanaka, S.; Chen, P. Welding Window: Comparison of Deribas' and Wittman's Approaches and SPH Simulation Results. Metals 2019, 9, 1323. [CrossRef]

2. Mahmood, Y.; Dai, K.; Chen, P.; Zhou, Q.; Bhatti, A.A.; Arab, A. Experimental and Numerical Study on Microstructure and Mechanical Properties of Ti-6Al-4V/Al-1060 Explosive Welding. Metals 2019, 9, 1189. [CrossRef]

3. Paul, H.; Chulist, R.; Mania, I. Structural Properties of Interfacial Layers in Tantalum to Stainless Steel Clad with Copper Interlayer Produced by Explosive Welding. Metals 2020, 10, 969. [CrossRef]

4. Carvalho, G.H.S.F.L.; Galvão, I.; Mendes, R.; Leal, R.M.; Loureiro, A. Aluminum-to-Steel Cladding by Explosive Welding. Metals 2020, 10, 1062. [CrossRef]

5. Inao, D.; Mori, A.; Tanaka, S.; Hokamoto, K. Explosive Welding of Thin Aluminum Plate onto Magnesium Alloy Plate Using a Gelatin Layer as a Pressure-Transmitting Medium. Metals 2020, 10, 106. [CrossRef]

6. Nishi, M.; Tanaka, S.; Vesenjak, M.; Ren, Z.; Hokamoto, K. Fabrication of Composite Unidirectional Cellular Metals by Using Explosive Compaction. Metals 2020, 10, 193. [CrossRef]

7. Su, S.; Chen, S.; Mao, Y.; Xiao, J.; Vivek, A.; Daehn, G. Joining Aluminium Alloy 5A06 to Stainless Steel 321 by Vaporizing Foil Actuators Welding with an Interlayer. Metals 2019, 9, 43. [CrossRef]

8. Wang, H.; Wang, Y. Characteristics of Flyer Velocity in Laser Impact Welding. Metals 2019, 9, 281. [CrossRef]

9. Emadinia, O.; Ramalho, A.M.; de Oliveira, I.V.; Taber, G.A.; Reis, A. Influence of Surface Preparation on the Interface of Al-Cu Joints Produced by Magnetic Pulse Welding. Metals 2020, 10, 997. [CrossRef]

10. Bellmann, J.; Lueg-Althoff, J.; Niessen, B.; Böhme, M.; Schumacher, E.; Beyer, E.; Leyens, C.; Tekkaya, A.E.; Groche, P.; Wagner, M.-X.; et al. Particle Ejection by Jetting and Related Effects in Impact Welding Processes. Metals 2020, 10, 1108. [CrossRef]

11. Niessen, B.; Schumacher, E.; Lueg-Althoff, J.; Bellmann, J.; Böhme, M.; Böhm, S.; Tekkaya, A.E.; Beyer, E.; Leyens, C.; Wagner, M.-X.; et al. Interface Formation during Collision Welding of Aluminum. Metals 2020, 10, 1202. [CrossRef]

12. Wang, H.; Wang, Y. High-Velocity Impact Welding Process: A Review. Metals 2019, 9, 144. [CrossRef]

Publisher's Note: MDPI stays neutral with regard to jurisdictional claims in published maps and institutional affiliations.

(C) 2020 by the authors. Licensee MDPI, Basel, Switzerland. This article is an open access article distributed under the terms and conditions of the Creative Commons Attribution (CC BY) license (http://creativecommons.org/licenses/by/4.0/). 